\title{
A Search for GeV-TeV Emission from GRBs Using the Milagro Detector
}

\author{
Taylor Aune for the Milagro Collaboration ${ }^{1}$ \\ Santa Cruz Institute for Particle Physics, University of California, Santa Cruz, 1156 High Street, Santa Cruz, \\ CA, 95060, USA
}

\begin{abstract}
The Milagro detector surveyed the sky almost continuously in the very high energy regime from January 2000 through March 2008. During that time, over 130 GRBs have been detected and well localized by satellites within its 2 sr field of view. We have used Milagro data to search for $>1 \mathrm{GeV}$ emission from these bursts. Milagro is a water Cerenkov detector designed primarily for observations in the 0.1-100 TeV energy range. Milagro was operated simultaneously in both standard mode and "scaler" mode. In the standard analysis, the direction and energy of an incoming particle is determined by reconstructing the air shower produced when the particle interacts in the Earth's atmosphere. In "scaler" mode, the rates of the PMTs are monitored and fluctuations temporally coincident with GRBs are searched for. No significant emission was discovered using either method and the upper limit on the emission for each burst with known redshift is reported.
\end{abstract}

Keywords: $\gamma$-ray, $\gamma$-ray bursts, $\gamma$-ray telescope

PACS: 95.85.Pw 98.70.Rz 95.55.Ka

\section{INTRODUCTION}

Milagro is ground-based gamma-ray observatory located in the Jemez mountains (2630 m a.s.l.) outside Los Alamos, New Mexico. The detector itself is a large pool of highly purified water, fit with a light-tight cover, and instrumented with 723 photomultiplier tubes (PMTs). Furthermore, an array of 175 water tanks, each 4000 liters and instrumented with a single PMT, is distributed around the pond, which serves to increase the effective area of the detector. Milagro is a member of the extensive air shower class of particle detectors, and as such, is primarily sensitive to photons in the $\mathrm{TeV}$ energy range. In addition to being operated in standard mode in which every air shower event is reconstructed, Milagro is also continuously operated using the "scaler" or "single-particle" technique $[1,2,3,4]$ in which statistically significant excesses in the PMT count rates, temporally coincident with satellite-detected GRBs, are searched for. These combined analyses allow for energy coverage ranging from $1 \mathrm{GeV}$ to over $100 \mathrm{TeV}$. Many GRB afterglow models $[5,6,7]$ predict production of photons in the GeV-TeV energy range and $\mathrm{GeV}$ emission has indeed been detected by both previous and current generation space-based $\gamma$-ray detectors [8, 9]. The spectral behavior of GRBs in the very high energy regime, however, remains largely unknown.

\section{STANDARD ANALYSIS}

For energies $>100 \mathrm{GeV}$, the Milagro standard analysis is used to search for an excess of events above the expected background, temporally and directionally coincident with satellite-detected GRBs. An estimate of the number of background events is made by characterizing the angular distribution of the background using 2 hours of data surrounding the burst, as described in [10]. The total number of events falling within a circular bin of radius $1.6^{\circ}$ centered on the burst is summed over the duration of the burst. The significance of the excess (or deficit) of each burst is evaluated using eq. (17) of $\mathrm{Li} \& \mathrm{Ma}$ [11]. Given the observed on-source counts and the predicted background, the 99\% confidence upper limits on signal counts is computed using the Feldman-Cousins prescription [12]. This upper

${ }^{1}$ A. A. Abdo, B. T. Allen, T. Aune, D. Berley, E. Blaufuss, S. Casanova, C. Chen, B. L. Dingus, R. W. Ellsworth, M. M. Gonzalez, J. A. Goodman, E. Hays, C. M. Hoffman, B. Hopper, P. H. Hüntemeyer, B. E. Kolterman, C. P. Lansdell, J. T. Linnemann, J. E. McEnery, A. I. Mincer, P. Nemethy, D. Noyes, J. M. Ryan, P. M. Saz Parkinson, A. Shoup, G. Sinnis, A. J. Smith, G. W. Sullivan, V. Vasileiou, G. P. Walker, D. A. Williams, X. W. Xu and G. B. Yodh 

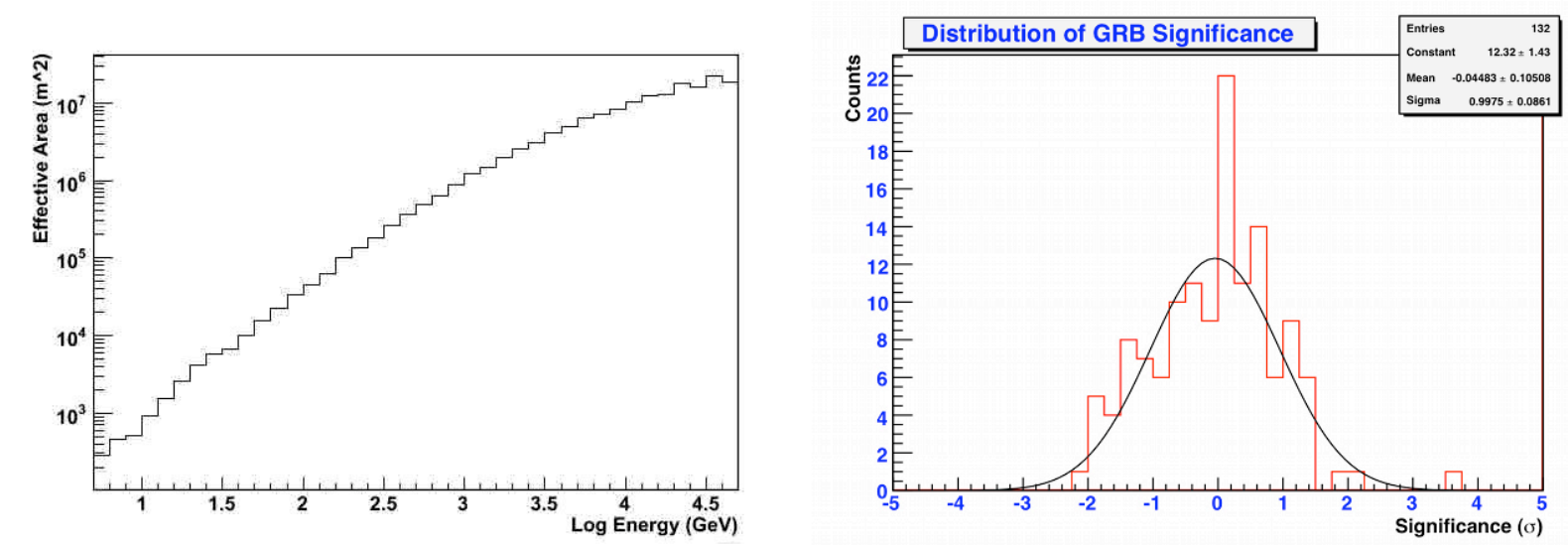

FIGURE 1. [Left] The effective area of the Milagro detector as a function of energy when operated in scaler mode. [Right] The histogram of significances of gamma-ray bursts in the Milagro FOV using the scaler method.

limit on the counts is then converted to an upper limit on the fluence, using the effective area of Milagro (see last section) and assuming a differential power-law photon spectrum.

\section{SCALER ANALYSIS}

In order to be sensitive to primary photons $<100 \mathrm{GeV}$, Milagro is operated in "scaler" mode. In scaler mode, the average PMT rate during the GRB is compared to the average rate during a period immediately before and after the burst. By doing the same for many comparable test intervals over an 11 day interval around the burst, it is seen that fluctuations are neither Poisson nor Gaussian. The excess (or deficit) rate during the GRB interval, relative to the background region, is compared to the distribution of excesses from the test intervals to obtain the significance of the excess and the 99\% confidence level upper limit on the rate. The resulting distribution of significances of the excess is shown in Figure 1, and is consistent with expectations from background fluctuations. The most significant excess is 3.5 standard deviations, with a $2.6 \%$ probability of occurring in a sample of 132 ; we don't interpret this as evidence for emission and report upper limits for all of the bursts.

\section{FLUENCE UPPER LIMITS}

The effective area of Milagro for gamma rays, is calculated using the standard Milagro detector simulation as described in $[10,13]$ while also accounting for any PMTs excluded due to excessive noise or other instrumental problems. A plot of the effective area of Milagro versus energy when operated in scaler mode is shown in Figure 1. An intrinsic GRB power law energy spectrum $\frac{d N}{d E} \sim E^{-2}$ for the scaler analysis and $\frac{d N}{d E} \sim E^{-2.4}$ for the standard analysis is assumed. This spectrum is then softened due to the gamma-rays being absorbed by interactions with the extragalactic background light (EBL) according to the model of [14]. For the bursts with measured or tentative redshifts, we report limits using EBL absorption for that redshift. No significant VHE emission was detected using either the scaler or standard analyses. For the scaler method, the fluence upper limits (at the $99 \%$ confidence level) between 1 and $100 \mathrm{GeV}$ for the unabsorbed power law spectrum are given in Table 1, along with the fluence upper limits $>100 \mathrm{GeV}$ obtained using the standard analysis.

We acknowledge Scott Delay and Michael Schneider for their dedicated efforts in the construction and maintenance of the Milagro experiment. This work has been supported by the National Science Foundation (under grants PHY0245234, -0302000, -0400424, -0504201, -0601080, and ATM-0002744) the US Department of Energy (Office of High-Energy Physics and Office of Nuclear Physics), Los Alamos National Laboratory, the University of California, and the Institute of Geophysics and Planetary Physics. 
TABLE 1. Gamma-ray bursts with known redshift in Milagro's field of view from January, 2000 to March, 2008. $\theta$ is the zenith angle of the source in the FOV of Milagro. 99\% confidence-limit scaler upper limits are given for both the scaler and standard analysis methods. Only upper limits for $z<1$ were calculated using the standard analysis method, since at larger redshifts $>100 \mathrm{GeV}$ photons are severely attenuated. For the scaler method, limits are only reported for $\mathrm{z}<3$.

\begin{tabular}{ccccccc}
\hline GRB & $T_{90}(\mathrm{~s})$ & $\theta(\mathrm{deg})$ & $z$ & scaler $\sigma$ & scaler UL $\left(\mathrm{ergs} / \mathrm{cm}^{2}\right)$ & standard UL $\left(\mathrm{ergs} / \mathrm{cm}^{2}\right)$ \\
\hline $000301 \mathrm{c}$ & 14 & 37.6 & 2.03 & 0.0 & $1.3 \mathrm{e}-3$ & - \\
000926 & 25 & 15.9 & 2.04 & -1.2 & $2.7 \mathrm{e}-4$ & - \\
010921 & 25 & 10.4 & 0.45 & 0.6 & $3.0 \mathrm{e}-4$ & $2.9 \mathrm{e}-4$ \\
021211 & 6 & 34.8 & 1.01 & 0.3 & $7.0 \mathrm{e}-5$ & - \\
040924 & 1 & 43.3 & 0.859 & -0.5 & $1.2 \mathrm{e}-4$ & $-4 \mathrm{e}-3$ \\
050319 & 15 & 45.1 & 3.24 & 1.3 & - & - \\
050502 & 20 & 42.7 & 3.793 & -1.1 & - & - \\
050505 & 60 & 28.9 & 4.3 & -2.0 & - & $1.1 \mathrm{e}-6$ \\
$050509 \mathrm{~b}$ & 1 & 10.0 & $0.226 ?$ & -0.5 & $3.3 \mathrm{e}-6$ & - \\
050820 & 20 & 21.9 & 2.612 & -0.3 & $7.3 \mathrm{e}-4$ & $4.2 \mathrm{e}-6$ \\
051103 & 1 & 49.9 & $0.001 ?$ & 0.5 & $2.1 \mathrm{e}-5$ & - \\
051109 & 36 & 9.6 & 2.346 & 1.2 & $1.4 \mathrm{e}-3$ & - \\
051111 & 20 & 43.7 & 1.55 & 0.1 & $2.7 \mathrm{e}-3$ & - \\
051221 & 2 & 41.8 & 0.5465 & 0.2 & $1.2 \mathrm{e}-4$ & - \\
060210 & 5 & 43.4 & 3.91 & -0.6 & - & - \\
060218 & 2000 & 43.7 & 0.0331 & 0.0 & $9.7 \mathrm{e}-2$ & - \\
$060510 \mathrm{~b}$ & 300 & 42.8 & 4.9 & -0.8 & - & - \\
060906 & 43 & 28.8 & 3.685 & -0.2 & - & - \\
061210 & 1 & 23.4 & $0.41 ?$ & 1.2 & $1.7 \mathrm{e}-5$ & - \\
070125 & 60 & 9.5 & 1.547 & -0.2 & $4.9 \mathrm{e}-4$ & - \\
070208 & 48 & 31.7 & 1.165 & -0.2 & $3.6 \mathrm{e}-4$ & - \\
070521 & 60 & 8.7 & $0.55 ?$ & 1.2 & $6.8 \mathrm{e}-4$ & - \\
070529 & 120 & 44.7 & 2.4996 & -0.1 & $2.7 \mathrm{e}-1$ & - \\
071122 & 69 & 44.0 & 1.14 & -0.5 & $1.4 \mathrm{e}-2$ & - \\
080310 & 365 & 44.9 & 2.42 & 1.0 & $1.5 \mathrm{e}-1$ & - \\
$080319 \mathrm{~b}$ & 50 & 43.2 & 0.937 & -2.0 & $1.9 \mathrm{e}-3$ & $5.3 \mathrm{e}-4$ \\
$080319 \mathrm{c}$ & 20 & 19.6 & 1.95 & 0.4 & $1.4 \mathrm{e}-3$ & \\
080330 & 60 & 27.8 & 1.51 & 0.0 & & - \\
\hline
\end{tabular}

\section{REFERENCES}

1. S. O'Brian, and N. A. Porter, Astrophys. Space Sci. 42, 73-76 (1976).

2. C. Morello, G. Navarra, and L. Periale, Nuovo Cimento 7C, 682-688 (1984).

3. M. Aglietta et al., Astrophys. J. 469, 305-310 (1996).

4. S. Vernetto, Astropart. Phys. 13, 75-86 (2000).

5. B. Zhang, and P. Meszaros, Astrophys. J. 559, 110-112 (2001).

6. A. Pe'er, and E. Waxman, Astrophys. J. Lett. 603, L1-L4 (2004).

7. X. Y. Wang et al., Astrophys. J. Lett. 546, L33-L37 (2001).

8. K. Hurley et al., Nature 372, 652 (1994).

9. F. Piron, "High-Energy emission tail in Gamma-Ray Bursts observed by the Fermi Large Area Telescope," in Proceedings of the Huntsville Gamma-Ray Burst Symposium, 2008.

10. R. Atkins et al., Astrophys. J. 595, 803 (2003).

11. T. P. Li, and Y. Q. Ma, Astrophys. J. 272, 317L (1983).

12. G. J. Feldman, and R. D. Cousins, Phys. Rev. D 57, 3873 (1998).

13. R. Atkins et al., Astrophys. J. 630, 996 (2005).

14. J. R. Primack et al., "Observational Gamma-ray Cosmology," in Proc. 2nd International Symposium on High Energy Gamma-Ray Astronomy, edited by F. A. Aharonian, Heidelberg, Germany, 2005, vol. 745. 